\title{
Selbstreflexives Schreiben
}

\section{Hildegard Keller}

Professorin für Deutsche Literatur, Universität Zürich, Visiting Professor, Indiana University, Bloomington, CEO Bloomlight Productions

Stellen Sie sich gelegentlich Fragen zu Ihrem ärztlichen Handeln? Wenden Sie sich schreibend an den inneren Anderen und ermöglichen so einen Dialog mit sich selbst? Selbstreflexives Schreiben ist Selbstzuwendung mit grosser Wirkung, stärkt Ihre intrinsische Motivation und Resilienz, und ist ein Weg, Ihre Würde und Autonomie als ärztlich Tätige zu leben.

«Ich verstehe die Geschichte nur von dem Boden aus, auf dem ich selbst stehe», schrieb Hannah Arendt, als sie 20 war. Viel später forderte sie den Dialog mit sich selbst, nachdem sie als Gerichtsreporterin in Jerusalem Adolf Eichmann sprechen und schweigen gehört hatte. Eichmann, so Arendt, kannte keine andere Sprache als "Amtsdeutsch" und hatte sich erlaubt, wie ein «job-holder» zu gehorchen, ohne den leisesten Schimmer vom Dialog mit sich selbst. Arendts Fazit war pragmatisch: Ein Mensch muss sich das Selber-Denken leisten, damit er mit sich selbst ein Leben lang zusammenleben kann. Im Schreibworkshop für Ärztinnen und Ärzte, den ich in Partnerschaft mit dem Center for Medical Humanities am Institut für Biomedizinische Ethik und Medizingeschichte der Universität Zürich durchführe, gebe ich Hannah Arendt, vor allem aber Ihnen das Wort.

Ich schreibe diese Zeilen in Rom, wo ich nach zwei Frauen forsche. Hier pflegte die grosse Schriftstellerin Ingeborg Bachmann den Dialog mit sich selbst und legte nach der Trennung von Max Frisch ihre poetische Krankenakte an. Im Schreibworkshop bekommt auch Ingeborg Bachmann mit ihrer Rede an die Ärzte das Wort: «Bitte seien Sie, was Sie sein können, nämlich Ärzte.»

Lydia Welti-Escher, die Kunstmäzenin aus Zürich, wurde unter Vorspiegelung falscher Tatsachen und einem mutwillig geäusserten Verdacht des Wahnsinns in der Römer Psychiatrie interniert. Die sorgfältige Anamnese der italienischen Psychiater scheint mir das unwahrscheinlichste Glück für Lydia. Die Ärzte stellten Fragen, hörten tagelang zu und zeichneten Lydias Selbstreflexionen minuziös auf, auch Zitate aus ihren in der Klinik notierten Gedanken einer Frau. Lydia entdeckte unter misslichen Umständen ihre Auto- nomie, ihre Freude am familiensystemischen Erzählen, und ihre Lust, über Gott und die Welt und die Liebe nachzudenken. Es grenzt an ein Wunder, dass ihr Originalton durch die italienischen Psychiater überliefert wurde. Im Schreibworkshop für Ärztinnen und Ärzte bekommt auch Lydia das Wort. Wir sind neugierig auf Sie.

\section{Essay-Wettbewerb: \\ Premio Pusterla Senior 2020}

Das Center for Medical Humanities am Institut für Biomedizinische Ethik und Medizingeschichte der Universität Zürich vergibt im Mai 2020 zum zweiten Mal den Essay-Preis Premio Pusterla Medizinethik Senior. Der Preis wird vergeben für den besten Essay, der ethische Probleme und Konfliktsituationen in der ärztlichen Praxis und Klinik in literarisch ansprechender Form verarbeitet. Der Preis ist benannt nach Dr. med. Edio Pusterla, der den Wettbewerb mit einer grosszügigen Zuwendung ermöglicht hat.

\section{Bedingungen:}

- Text auf Deutsch

- Textlänge max. 12000 Zeichen (inkl. Leerzeichen)

- Nur bisher unveröffentlichte Texte zugelassen

- Text als PDF-Datei an biomed[at]ibme.uzh.ch

- Einsendeschluss: 31.3.2020

- Bisherige GewinnerInnen können nicht am Wettbewerb teilnehmen

Weitere Informationen: www.ibme.uzh.ch

\section{Workshop: Selbstreflexives Schreiben}

\section{mit Hildegard Keller}

Der Workshop gibt Impulse zur ethischen Reflexion von ärztlicher Erfahrung, Redaktion im Lerntandem und individuelles Coaching und eignet sich als Begleitung zurTeilnahme am Premio Pusterla Medizinethik Senior, ist aber als Fortbildung für Ärztinnen und

Ärzte davon unabhängig.

- 28.2.2020/13.3.2020/27.3.2020, je 12-13.30 Uhr,

Universität Zürich

- Max. 20 Teilnehmerlnnen ohne/mit Facharzttitel, 7 SIWF-Credits beantragt

Anmeldung bis 1.2.2020: info[at]bloomlightproductions.ch

Weitere Informationen:

www.bloomlightproductions.ch und 0443822153 